\title{
Medical Physics Contributes to The Advancement in Medicine
}

\author{
Kwan Hoong $\mathrm{Ng}^{1 *}$, Jeannie Hsiu Ding Wong ${ }^{1}$, Chai Hong Yeong ${ }^{2}$, Hafiz Mohd Zin³ and Noriah Jamal ${ }^{1}$ \\ ${ }^{1}$ Department of Biomedical Imaging, Faculty of Medicine, University of Malaya, Kuala Lumpur, Malaysia \\ ${ }^{2}$ School of Medicine, Faculty of Health and Medical Sciences, Taylor's University, Malaysia \\ ${ }^{3}$ Advanced Medical and Dental Institute, Universiti Sains Malaysia, Bertam, Malaysia
}

\begin{abstract}
Medical physics is the application of physics principles and techniques in medicine. Medical physicists are actively applying their knowledge and skills in the prevention, diagnosis and treatment of diseases to improve health via research and clinical practice. In this paper, we present the roles of medical physicists in the three primary fields, namely, diagnostic imaging, radiotherapy and nuclear medicine. Medical physicists have been playing a crucial role in the advancement of new technologies that have revolutionised medicine today. This includes the continuous development of medical imaging and radiotherapy techniques since the discovery of Xray and radioactivity. The last decade has seen tremendous development in the field that allows for better diagnosis and targeted treatment of various diseases. In the era of big data and artificial intelligence, while medical physicists continue to ensure that the application of the technologies in medicine is optimal and safe, it is paramount for the profession to evolve and be equipped with new skills to continue to contribute to the advancement of medicine.
\end{abstract}

Keywords: radiology; nuclear medicine; radiation therapy; X-ray; magnetic resonance imaging

\section{INTRODUCTION}

Medical Physics is the application of physics principles and techniques in medicine. Medical physicists are actively applying their knowledge and skills in the prevention, diagnosis and treatment of diseases to improve health via research and clinical practice. Medical physicists have been playing a crucial role in the advancement of new technologies that have revolutionised medicine today. Medical physics can be classified into several specialities, such as diagnostic imaging physics, nuclear medicine physics, radiation oncology physics, medical health physics (radiation protection), non-ionising radiation physics and physiological measurement (IOMP, 2012; Ng et al., 2009; $\mathrm{Ng}$, 2008). Today's medical physicists are no longer working behind the scenes; they guide clinicians to improve patient care.

\section{THE ROLES OF MEDICAL PHYSICISTS}

The roles of medical physicists vary slightly in the three specialities. In radiotherapy, medical physicists are involved in "all procedures that ensure consistency of the medical prescription, and safe fulfilment of that prescription, i.e. dose to the target volume, together with minimal dose to normal tissue, minimal exposure of personnel and adequate patient monitoring aimed at determining the result of the treatment" (WHO, 1988). It is important to verify and guarantee that the dose is delivered as intended throughout treatment delivery. Various organisations, such as International Atomic Energy Agency (IAEA), European Society for Therapeutic Radiation Oncology (ESTRO), American Association of Physicists in Medicine (AAPM) and Institute of Physics and Engineering in Medicine (IPEM) have published recommendations for quality assurance (QA) standards in radiotherapy. The guidelines often underline the two aspects of tolerance level of uncertainties, i.e. the 
dose delivered, and the geometric accuracy of the dose planned. Because of the required level of consistency and accuracy, the success of radiotherapy treatment has been dependent on the strict practice of QA programme by the medical physicists in radiotherapy departments (Hizam et al., 2019).

Medical physicist uses dosimeters to verify the dose and spatial distributions of the planned radiation required. Various types of dosimeters are used for QA purposes throughout radiotherapy advancement, including ionisation chambers, diode detectors, film and electronic portal imaging device (EPID). The challenge is that the prospective dosimeter needs to be characterised and fully understood by the medical physicist before being used in radiotherapy QA (Zin et al., 2013).

In diagnostic imaging physics or radiological physics, medical physicists work with radiologists and radiographers to ensure optimised image quality and radiation dose is achieved. In radiotherapy physics, medical physicists work with radiation oncologists and radiation therapists to ensure optimal dose delivery to tumours while sparing the surrounding healthy tissues. A medical physicist often takes on the role as a radiation protection officer (RPO), which is defined as a technically competent person appointed to supervise the application of appropriate radiation protection regulations, measures and procedures. The key responsibility is to ensure radiation safety to the patients, staff and members of the public.

Traditionally medical physicists also play several important roles in healthcare. For example, they provide technical advice on medical equipment specification, acquisition and verification process. They also assist in radiation shielding calculation, installation and commissioning of facilities. Medical physicists also assist in emergencies such as radioactive material spillage and radiological accidents.

\section{DIAGNOSTIC IMAGING}

Technological progress in computed tomography (CT) has enlarged its applications since its invention in 1971. Advances in hardware and software (image reconstruction and processing) have increased scanning speed with reduced radiation dose. During the last decade, there have been several innovations in CT technology that have greatly improved the imaging performance and dose efficiencies, such as multidetector-row $\mathrm{CT}$, iterative reconstruction algorithms, dual-source or dual-energy CT, cone-beam CT and phase-contrast CT (Tabari et al., 2017; Ginat \& Gupta, 2014). These advances enabled scanning up to 720 slice per rotation within half a second, leading to faster scans at higher image qualities, producing almost motion artefactfree volumetric images such as for cardiac imaging (Figure 1).

CT scanner is also one of the modalities that has moved into hybrid imaging. For example, in PET-CT whereby CT is combined with positron emission tomography (PET) which is an excellent tool for cancer detection and staging. This technology combines anatomic imaging of CT with functional imaging of PET and angio CT. While low dose fluoroscopy of the angiography system provides real-time visual of the manoeuvring of the catheters in the blood vessels, CT provides a $3 \mathrm{D}$ reconstructed image of the human anatomy. The fusion of these two technologies is akin to having a $3 \mathrm{D}$ map of the tumour vascularization system, providing interventional radiologists with excellent navigational imaging tool for more accurate positioning of the catheters and drug deposition in the tumour.

Palpation is an ancient technique practised by medical practitioners. A tumour may feel harder or stiffer. Clinically, it is well known that histology of the tissue affects its mechanical properties, sometimes manifested as increased fibrosis in the parenchyma (Leong et al., 2018). However, if the tumour is deep-seated, this manual technique would not be practical. Ultrasound elastography uses shear wave elastography techniques to evaluate tissue stiffness. A shortduration acoustic 'push' pulse is used to generate shear waves in real-time that propagate perpendicular to the main ultrasound beam. When the waves arrive at the targeted tissue, the tissue is 'pushed' in the direction of propagation, causing it to displace temporarily (Leong et al., 2018). Research has shown that shear wave elastography is becoming a useful tool to diagnose end-stage chronic kidney disease (Leong et. al., 2019; Leong et al., 2018). 


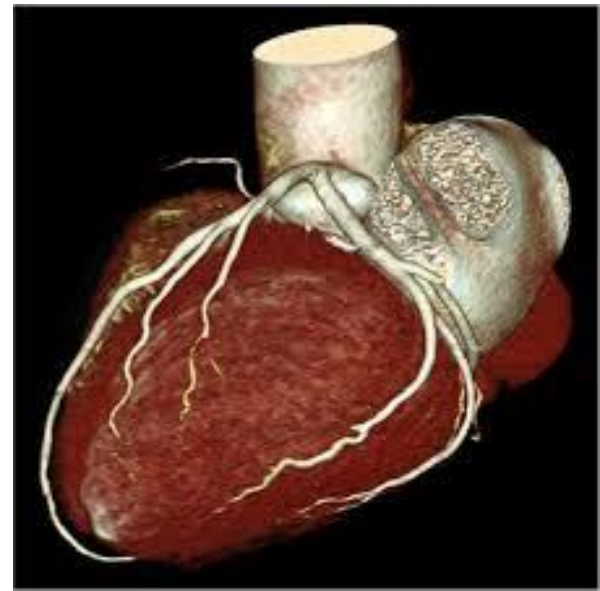

Figure 1. Cardiac CT image for diagnosis of heart and blood vessels disorder (Courtesy of Assoc. Prof. Dr Raja Rizal)

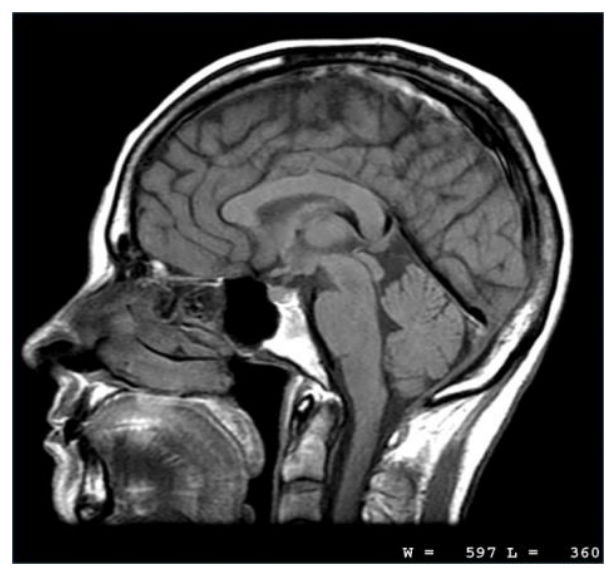

Figure 2. MRI image of the brain. (Courtesy of Dr Azlan Che Ahmad, University of Malaya)

Magnetic resonance imaging (MRI) is one of the most versatile imaging modalities for acquiring a high-resolution image to provide diagnostic information for systems such as cardiovascular, musculoskeletal, neurological and others (Figure 2). MRI measures the spatial distribution of particular nuclear spins of protons in the tissue. In nuclear magnetic resonance (NMR), the protons precess at a specific frequency known as the Larmor frequency. Each type of nucleus has a specific Larmor frequency. Electrical signals from the spins are measured after they are excited by the radiofrequency $(\mathrm{RF})$ pulses irradiated in a static magnetic field. The NMR signal itself carries no spatial information. The spatial information that is needed to generate an image is provided by the magnetic field gradients generated by the gradient coils.

Advanced techniques such as functional MRI (fMRI) and magnetic resonance spectroscopy (MRS) enable visualisation and mapping of activity of task-specific neuronal networks, which has opened up significant clinical applications in neuroscience, functional neurosurgery and psychiatry. Although clinical MRI is most commonly performed at 1.5 $\mathrm{T}$ and 3.0 $\mathrm{T}$, higher field strength such as 7 $\mathrm{T}$ for research purposes is becoming more popular because of their increased sensitivity and resolution.

\section{RADIOTHERAPY}

Shortly after Wilhelm Röntgen discovered X-ray in 1896, it has been used for cancer treatment. Both kilovoltage and orthovoltage X-ray teletherapy techniques were used in the early days. High energy teletherapy based on the gamma rays emitted from Cobalt-60 was developed in the 1950s. It has been the work-horse of radiotherapy for decades until it was superseded by linear accelerator that accelerates electron to produce megavoltage (MV) energy X-ray to destroy the tumour cells. The MV energy X-rays produced penetrate further into the body and cause less damage to the skin.

Development of intensity-modulated radiation therapy (IMRT) during the last quarter of the 2oth century has improved the precision and accuracy of delivering high radiation dose to the target. IMRT delivers high-energy radiation by collimating the radiation with a beam shaping system called a multi-leaf collimator (MLC) to conform to the geometry of the tumour (Webb, 2003). The aim is to deliver the radiation to the target and minimise the exposure to the surrounding healthy tissue and other organs at risk. Figure 3 illustrates the resulted dose distribution from conformal radiotherapy and advanced radiotherapy. To achieve this high level of conformality, the field geometry and dose rate must be varied in real-time surrounding the patient. 


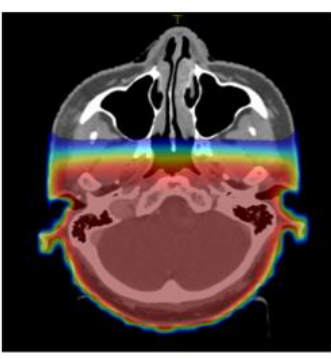

3DCRT

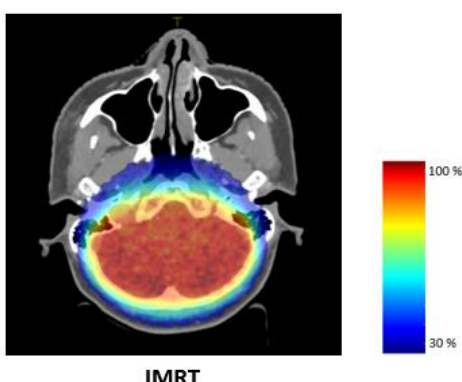

IMRT
Figure 3. IMRT technique can achieve better dose conformality to the brain and better dose sparing to other healthy tissues compared with $3 \mathrm{D}$ conventional radiotherapy (3DCRT)

Proton therapy, the latest advanced radiation therapy uses a high-energy proton beam (about 70 to $240 \mathrm{MeV}$ ) with precision to deliver the radiation dose to the tumour and spare the surrounding healthy tissues (Levin et al., 2005). This form of treatment is appropriate for treating tumours near to the sense organs, such as the eye, brain, and spinal cord.

As the proton beam enters the patient's body, the absorbed dose increases very slowly with increasing depth, then abruptly rising to a peak when the proton is ultimately stopped and deposit its maximum energy to the target. This is known as the Bragg peak. The characteristics of the proton beam can be determined precisely, and the beam can be directed so the Bragg peak occurs exactly within the tumour site as shown in Figure 4.

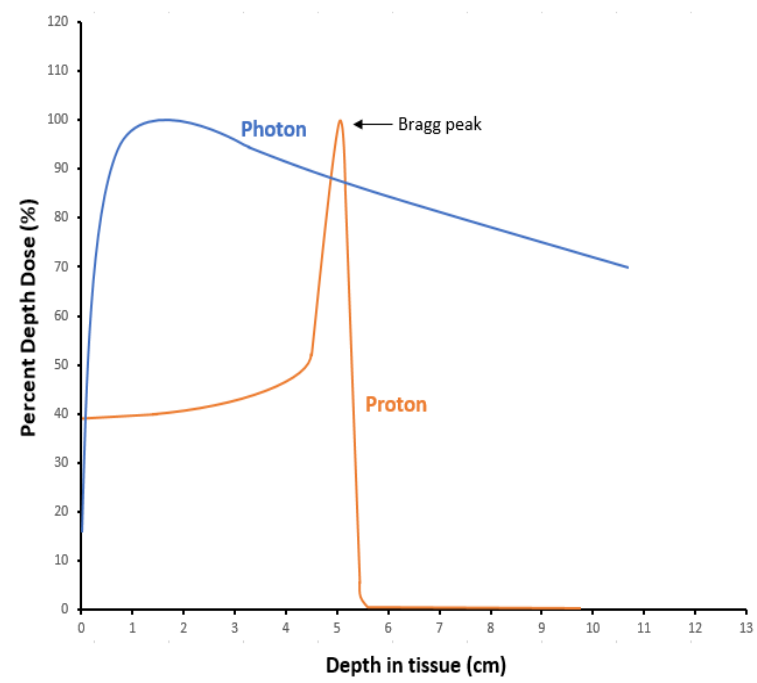

Figure 4. Depth dose data highlighting the Bragg peak for 80 $\mathrm{MeV}$ proton compared to $6 \mathrm{MV}$ photon
Advanced radiotherapy provides more targeted radiation delivery to the cancerous tissues compared to the conventional technique. However, these treatments tend to have introduced errors in delivery due to uncertainties in the target location citation needed. Errors in radiation delivery may also result from patient movement during treatment, including patient positioning during treatment and organ motion due to breathing. Advancement in imaging technologies has also aided in improving the delivery of radiation through these advanced techniques. The beam delivery can also be gated, i.e. turned on and off, depending on the tracked tumour motion due to physiological organ motion such as breathing, so that the beam is delivered only when the tumour is within the beam aperture (Briere et. al., 2009; Tang et al., 2009). The complexity of radiotherapy treatment demands a chain of quality assurance (QA) processes before the final treatment delivery to the patient to ensure treatment success. Otherwise, the patients will suffer from tumour recurrence and unnecessary radiation side effects.

These new challenges of radiotherapy and the resulted risks are being safeguarded by the medical physicists. This is to minimise treatment errors so that radiotherapy can be delivered precisely, accurately and optimally.

\section{NUCLEAR MEDICINE}

Nuclear medicine is a medical speciality that uses radioisotopes for diagnosis and treatment of diseases. During the procedure, a known amount of radiopharmaceutical (a radioisotope labelled with a biological carrier) is administered to the patient's body via injection, ingestion or inhalation. Unlike X-ray imaging, nuclear medicine imaging detects the gamma radiation emitting from the radioisotope uptake within the body rather than radiation that is generated by the X-ray source. Besides, nuclear medicine imaging elucidates the physiological function of an organ rather than its anatomy. It is often combined with CT or MRI findings to assist a clinical diagnosis.

Advanced technologies in both hardware and software have enabled hybrid imaging to be carried out. For examples, a gamma camera instrument is combined with a CT scanner to produce single-photon emission computed 
tomography SPECT-CT images, PET-CT or MRI scanner to produce PET-MR images. The fusion imaging technique provides more accurate information on both anatomy and physiology in a single study. Medical physicists ensure the quality and accuracy of image production and registration. Systematic and periodical quality control need to be included in the standard operating procedures (SOP) of a nuclear medicine department.

The recent development in radiopharmaceutical has also led to more advanced imaging and treatment of various diseases at the molecular level. As an example, peptide receptor radionuclide therapy (PRRT) using ${ }^{\circ} \mathrm{Y}$-DOTATOC or ${ }^{177 \mathrm{Lu}-D O T A T A C}$ (a radiolabelled somatostatin receptor) has shown very promising results in treating neuroendocrine tumours (NETs). The treatment involves the systemic administration of a radiolabelled peptide developed to target with high affinity and specificity receptors overexpressed on neuroendocrine tumours (Bodei et al., 2013). 177Lu-PSMA (prostate-specific membrane antigen) is another good example of radioimmunotherapy (RIT) that has shown good response rate and low toxicity in treating prostate cancer (Rahbar et al., 2018). The basic principle of RIT is illustrated in figure 5. The monoclonal antibody will bind specifically to an antigen associated with tumour increases the radiation dose delivered to the tumour while decreasing the dose to healthy tissues (Yeong et al., 2014).

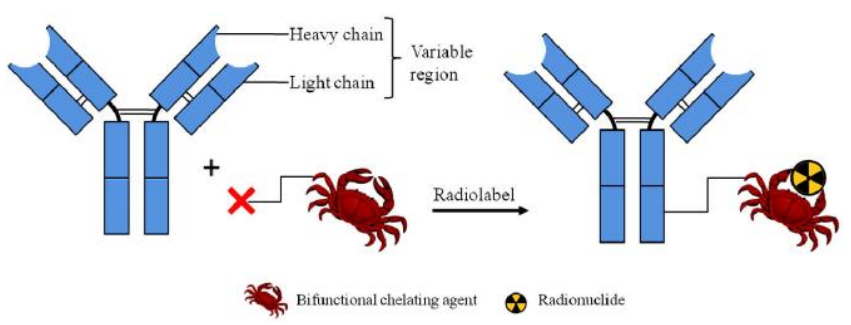

Figure 5. Basic principle of RIT. The monoclonal antibody is conjugated with a bifunctional chelating agent to bind the metallic radionuclides and to hold the reactive functional group (X) that binds to the $\mathrm{N}$-terminal and $\varepsilon$-amines from lysines on the antibody, adapted from (Yeong et al., 2014)

Theranostics is a new area of nuclear medicine which is expanding to provide therapeutic as well as diagnostic imaging using the same radiopharmaceutical formulation. Theranostics is derived from the Greek words "therapy" and "diagnosis" (Frangos and Buscombe, 2019). Theranostics is paving the way towards personalised or precision medicine. It uses molecular targeting vectors (eg. peptides) labelled with diagnostic and therapeutic radionuclide(s), to acquire diagnostic images as well as to deliver a therapeutic radiation dose to the target. This can be achieved by either using a therapeutic radionuclide that also emits radiation for

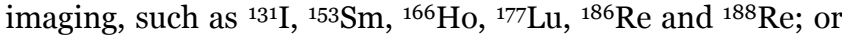
incorporating two radionuclides (one for imaging and another for therapy) into the same formulation. The diagnostic imaging provides further information on the disease, its status and the characteristics of a patient. This information allows the decision to be made on the quantity, timing, type of drugs and choice of treatment procedures for the specific patient, as well as to monitor early response to treatment and predict efficacy.

Theranostics started as early as the 1940's when radioiodine ( $\left.{ }^{131} \mathrm{I}\right)$ was first used to diagnose and treat thyroid cancer (Herrmann et al., 2017). ${ }^{131}$ I emits both therapeutic beta and diagnostic gamma radiations which is a classic example of theranostics agent. ${ }^{131}$ I targets mainly the thyroid cells, to destroy those uptakes the iodine, without any side effects on the rest of the body. During in the last decade, the growth of theranostics treatments has been accelerating (Svenson, 2013). The popular theranostics radiopharmaceuticals that are FDA-approved or currently

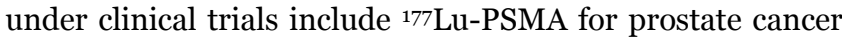
(Eiber et al., 2017), ${ }^{131} \mathrm{I}$-antibody or ${ }^{225 A c-a n t i b o d y ~ f o r ~}$ leukaemia (Moek et al., 2017), ${ }^{211} \mathrm{At}-\mathrm{MABG}$ for pheochromocytoma (Seo, 2019), ${ }^{89} \mathrm{Zr} /{ }^{\circ} \mathrm{Y}$-Ibritumomab Tiuxetan for lymphoma (Perk et al., 2006), and many more.

\section{THE EXPANDING ROLES OF PHYSICISTS IN MEDICINE}

In the time and age of big data, the priorities of healthcare systems have been shifting from the traditional focus on safety and regulatory compliance to include new priorities such as personalised medicine, health economics and artificial intelligence (AI). Different research initiatives that focus on supporting the evidence-based use of technology and data analytics have emerged.

The latest Australasian College of Physical Scientists and Engineers in Medicine (ACPSEM) Position Statement (2019) 
highlighted the expanding and changing roles of medical physicists, amongst others (ACPSEM, 2018) :

- In radiology and imaging, optimisation of the use of ionising radiation now includes focusing on both the acquisition of images and the adaptation of computer-aided diagnostic tools and techniques in support of the clinical team and improved clinical results.

- Utilising scientific and quantitative skills to develop niche opportunities which focus on analysing and optimising clinical outcomes in areas such as orthopaedics, cardiac surgery and interventional cardiology.

- Engaging with opportunities offered by AI and machine learning as well as linking and developing datasets to inform clinical practice.
- Leading collaborative work focusing on radiation therapy such as MR-Linac technology development and proton therapy centre planning.

- On-site synthesis of both radionuclides and radiopharmaceuticals for diagnostic and therapeutic use.

\section{CONCLUSION}

While medical physics continue to support medicine in its traditional fields, the needs and urgency to evolve to adapt and contribute to the future of medicine are undeniable. Towards that end, curricula and training of medical physicists should be relooked into to prepare future generations of medical physicists with the necessary skills to address the challenges of the new era.

\section{REFERENCES}

ACPSEM 2018, ACPSEM position statement the role of physicists, scientists and engineers in medicine in Australasia.

https://www.acpsem.org.au/documents/item/18.

Bodei, L, Mueller-Brand, J, Baum, RP, Pavel, ME, Horsch, D, O'Dorisio, MS, O'Dorisio, TM, Howe, JR, Cremonesi, M, Kwekkeboom, DJ \& Zaknun, JJ 2013, 'The joint IAEA, EANM, and SNMMI practical guidance on peptide receptor radionuclide therapy (PRRNT) in neuroendocrine tumours', European Journal of Nuclear Medicine and Molecular Imaging, vol. 40, no. 5, pp. 800-816.

Briere, TM, Beddar, S, Balter, P, Murthy, R, Gupta, S, Nelson, C, Starkschall, G, Gillin, MT \& Krishnan, S 2009, 'Respiratory gating with EPID-based verification: the MDACC experience', Physics in Medicine \& Biology, vol. 54, no. 11, pp.3379.

Eiber, M, Fendler, WP, Rowe, SP, Calais, J, Hofman, MS, Maurer, T, Schwarzenboeck, SM, Kratowchil, C, Herrmann, K \& Giesel, FL 2017, 'Prostate-specific membrane antigen ligands for imaging and therapy', Journal of Nuclear Medicine, vol. 58 (Supplement 2), pp. 67S-76S.

Frangos, S \& Buscombe, JR 2019, 'Why should we be concerned about a “g”?', European Journal of Nuclear
Medicine and Molecular Imaging, vol. 46, no. 2, pp. 519519 .

Ginat, DT \& Gupta, R 2014, 'Advances in computed tomography imaging technology', Annual Review of Biomedical Engineering, vol. 16, pp. 431-453.

Herrmann, K, Larson, SM \& Weber, WA 2017, 'Theranostic concepts: more than just a fashion trend-introduction and overview', Journal of Nuclear Medicine, vol. 58 (Supplement 2), pp. 1S-2S.

Hizam, NDA, Ung, NM, Jong, WL, Zin, HM, Rahman, ATA, Loh, JPY \& Ng, KH 2019, 'Medical Physics Aspects of Intensity-Modulated Radiotherapy Practice in Malaysia', Physica Medica, vol. 67, pp. 34-39.

IOMP 2012, 'Role and Responsibilities', in IOMP Policy Statement No. 1, The Medical Physicist, May 2012, Beijing, China.

Leong, SS, Wong, JHD, Shah, MNM, Vijayananthan, A, Jalalonmuhali, M \& Ng, KH 2019, 'Comparison of shear wave elastography and conventional ultrasound in assessing kidney function as measured using $51 \mathrm{Cr}$ ethylenediaminetetraacetic acid and 99Tcdimercaptosuccinic acid', Ultrasound in Medicine \& Biology, vol. 45, no. 6, pp. 1417-1426.

Leong, SS, Wong, JHD, Md Shah, MN, Vijayananthan, A, Jalalonmuhali, M \& Ng, KH 2018, 'Shear wave 
elastography in the evaluation of renal parenchymal stiffness in patients with chronic kidney disease', The British Journal of Radiology, vol. 91, no. 1089, pp. 20180235

Levin, WP, Kooy, H, Loeffler, JS \& DeLaney, TF 2005, 'Proton beam therapy', British Journal in Cancer, vol. 93, pp. 849-854.

Moek, KL, Giesen, D, Kok, IC, de Groot, DJA, Jalving, M, Fehrmann, RS, Lub-de Hooge, MN, Brouwers, AH \& de Vries, EG 2017, 'Theranostics using antibodies and antibody-related therapeutics', Journal of Nuclear Medicine, vol. 58 (Supplement 2), pp. 83S-90S.

$\mathrm{Ng}, \mathrm{KH}$ 2008, 'Medical physics in 2020: will we still be relevant?' Australasian Physics \& Engineering Sciences in Medicine, vol. 31, no. 2, pp. 85-89.

$\mathrm{Ng}$, KH, Cheung, $\mathrm{KY}, \mathrm{Hu}, \mathrm{YM}$, Inamura, K, Kim, HJ, Krisanachinda, A, Leung, J, Pradhan, AS, Round, H, van Dorn, T \& Wong, TJ 2009, 'The role, responsibilities and status of the clinical medical physicist in AFOMP', Australasian Physical \& Engineering Sciences in Medicine, vol. 32, no. 4, pp. 175-179.

Perk, LR, Visser, OJ, Stigter-van Walsum, M, Vosjan, MJ, Visser, GW, Zijlstra, JM, Huijgens, PC \& Van Dongen, GA 2006, 'Preparation and evaluation of $89 \mathrm{Zr}$-Zevalin for monitoring of $90 \mathrm{Y}$-Zevalin biodistribution with positron emission tomography', European Journal of Nuclear Medicine and Molecular Imaging, vol. 33, no. 11, pp. 13371345 .

Rahbar, K, Afshar-Oromieh, A, Jadvar, H \& Ahmadzadehfar, H. 2018, 'PSMA theranostics: current status and future directions', Molecular imaging, vol. 17, pp. 1-9.

Seo, Y 2019, 'Quantitative imaging of alpha-emitting therapeutic radiopharmaceuticals', Nuclear Medicine and Molecular Imaging, vol. 53, no. 3, pp. 182-188.

Svenson, S 2013, 'Theranostics: are we there yet?', Molecular Pharmaceutics, vol. 10, no. 3, pp. 848-856.

Tabari, A, Lo Gullo, R, Murugan, V, Otrakji, A, Digumarthy, S \& Kalra, M 2017, 'Recent advances in computed tomographic technology', Journal of Thoracic Imaging, vol. 32, no. 2, pp. 89-100.

Tang, X, Lin, T \& Jiang, S 2009, 'A feasibility study of treatment verification using EPID cine images for hypofractionated lung radiotherapy', Physics in Medicine \& Biology, vol. 54, no. 18, pp. S1.

Webb, S 2003, 'The physical basis of IMRT and inverse planning, The British Journal of Radiology, vol. 76, no. 910, pp. 678-689.
World Health Organization (WHO) 1988, Quality Assurance in Radiotherapy, Geneva, Switzerland.

Yeong, $\mathrm{CH}$, Cheng, $\mathrm{MH} \& \mathrm{Ng}, \mathrm{KH}$ 2014, 'Therapeutic radionuclides in nuclear medicine: current and future prospects', Journal of Zhejiang University SCIENCE B, vol. 15 , no. 10 , pp. $845-863$.

Zin, HM, Harris, EJ, Osmond, JP, Allinson, NM \& Evans, PM 2013, 'Towards real-time VMAT verification using a prototype, high-speed CMOS active pixel sensor', Physics in Medicine \& Biology, vol. 58, no. 10, pp. 3359. 